\title{
Marmara Bölgesi'ndeki bazı mermer örneklerinde radyolojik tehlikelerin ve yaşam boyu kanser riskinin değerlendirilmesi
}

\author{
Kadir GÜNOĞLU* \\ Isparta Uygulamalı Bilimler Üniversitesi, Teknik Bilimler Meslek Yüksekokulu, Isparta. \\ Geliş Tarihi (Recived Date): 16.08.2018 \\ Kabul Tarihi (Accepted Date): 18.10 .2018
}

\section{Özet}

Bu çalışmada, Marmara Bölgesi' nden çıkarllan bazı mermerlerdeki doğal radyoaktivite seviyelerini belirlemek için ${ }^{226} \mathrm{Ra},{ }^{232} \mathrm{Th} v e{ }^{40} \mathrm{~K}$ konsantrasyonlarl gama spektrometre sistemi ile ölçülmüştür. Mermer numunelerindeki ${ }^{226} \mathrm{Ra},{ }^{232} \mathrm{Th}$ ve ${ }^{40} \mathrm{~K}$ ortalama aktivite konsantrasyonlarl, sirastyla 37.446, 34.593 ve $248.041 \mathrm{~Bq} / \mathrm{kg}$ olarak ölçülmüştür. Ölçümler sonucunda elde edilen ${ }^{226} \mathrm{Ra},{ }^{232} \mathrm{Th},{ }^{40} \mathrm{~K}$ aktivite konsantrasyonları kullanılarak soğurulan gama doz hızı (D), yıllık etkin doz eşdeğeri (YEDE), radyum eşdeğer aktivitesi $\left(R a_{e q}\right)$, iç tehlike endeksi $(H i)$ ve diş tehlike endeksi $(H d)$ değerleri hesaplanmıştır. Bunların yanı sıra, radyasyon maruziyetinin bir sonucu olan yaşam boyu kanser riski (LCR) hesaplanmiştır. Elde edilen tüm sonuçlar uluslararası kuruluşlar tarafindan önerilen limit değerler ile karşılaştırılmıştır. Bu sonuçlara göre, hem radyolojik zarar indeksleri açısından hem de doz parametreleri açısından hiç bir zarar teşkil etmediği sonucuna varılabilir.

Anahtar kelimeler: Doğal radyoaktivite, radyolojik tehlike, yaşam boyu kanser riski, mermer.

\section{Assessment of radiological hazards and lifetime cancer risk in some marble samples from the Marmara Region}

\begin{abstract}
In this study, ${ }^{226} \mathrm{Ra},{ }^{232} \mathrm{Th}$ and ${ }^{40} \mathrm{~K}$ concentrations were measured by gamma spectrometry system to determine the natural radioactivity levels in some marbles mining from the Marmara Region. Mean activity concentrations of ${ }^{226} \mathrm{Ra},{ }^{232} \mathrm{Th}$ and ${ }^{40} \mathrm{~K}$ in marble samples were measured as 37.446, 34.593 and $248.041 \mathrm{~Bq} / \mathrm{kg}$, respectively.
\end{abstract}

* Kadir GÜNOĞLU, kadirgunoglu@sdu.edu.tr, https://orcid.org/0000-0002-9008-9162 
The absorbed dose rate (D), annual effective dose equivalent(YEDE), radium equivalent activity $\left(\mathrm{Ra}_{\text {eq }}\right)$, internal hazard index $(\mathrm{Hi})$ and external hazard index $(\mathrm{Hd})$ values were calculated using the ${ }^{226} \mathrm{Ra},{ }^{232} \mathrm{Th},{ }^{40} \mathrm{~K}$ activity concentrations obtained as a result of the measurements. In addition, lifetime cancer risk (LCR), which is a consequence of radiation exposure, has been calculated. All the results obtained are compared with the limit values recommended by international organizations. According to these results, it can be concluded that there is no harm in terms of both radiological damage indices and dose parameters.

Keywords: Natural radioactivity, radiological hazard, lifetime cancer risk, marble.

\section{Giriș}

Dünyanın varoluşundan bu yana yeryüzünde yaşayan tüm canlılar doğal ve yapay radyasyona maruz kalmaktadırlar. Tüm yapı malzemeleri çoğunlukla kaya ve topraktan oluşur ve bu iki hammadde, doğal radyasyona sebep olan ${ }^{232} \mathrm{Th}$ ve ${ }^{238} \mathrm{U}$ bozunma serileri ve ${ }^{40} \mathrm{~K}$ doğal radyoaktif izotoplar içerir. Gama radyasyonu, ${ }^{40} \mathrm{~K}$ ve ${ }^{232} \mathrm{Th}$ ve ${ }^{238} \mathrm{U}$ serilerinden gelen radyonüklidler gibi doğal olarak oluşan radyoizotoplardan ve bunların tüm zemin oluşumlarında eser seviyelerinde bulunan bozunma ürünlerinden (karasal zemin radyasyonu olarak da adlandırılır) yayılır. Daha spesifik olarak, gama radyasyonuna bağlı doğal çevresel radyoaktivite, öncelikle çalışma yapılan bölgenin jeolojisine ve coğrafi koşullarına bağlıdır. Bu nedenle dünyanın her bölgesinde doğal radyoaktivite seviyelerinde farkl1lıklar gözlenebilir [1,2].

Yapı malzemelerindeki doğal radyonüklitler, hem doğrudan gama 1şımasından dış maruziyete hem de radon gazından kaynaklanan iç maruziyete neden olabilir. Duvarlardan, katlardan ve tavanlardan kaynaklanan gama radyasyonu, Radon, Thoron ve bunların bozunma ürünleri radyasyon maruziyetinin başlıca kaynaklarıdır. Yapı malzemelerinden gama 1şınlarının neden olduğu kapalı etkn dozun dünya çapında ortalama yılda yaklaşık $0.4 \mathrm{~m}$ Sv olduğu tahmin edilmektedir [3]. Bireyler zamanlarının \% 80'inden fazlasını bina içlerinde geçirdikleri için yapı malzemelerinden kaynaklanan iç ve dış radyasyona uzun süreli maruz kalma durumları yaratır.

Yapı malzemeleri doğal radyonüklid içerikleri nedeniyle iç mekânlarda önemli bir gama dozuna neden olabilir. Binalarda özellikle döşeme ve dekoratif amaçla granit, seramik fayans, mermerler vb. malzemelerin kullanımı dünya genelinde giderek artmaktadır [4]. Konut yapımında kullanılan bu tip malzemelerde bulunan doğal radyoaktivite miktarının bilinmesi önemlidir. Doğal olarak oluşan materyallerde aktivite konsantrasyonlarının belirlenmesi için bir dizi radyoanalitik yöntem kullanılabilir. Gama ışını spektrometreleri bu amaç için yaygın olarak kullanılmaktadır.

Dünya çapında yapılan çalışmalarda bazı taş ve yapı malzemelerindeki ${ }^{226} \mathrm{Ra},{ }^{232} \mathrm{Th}$ ve ${ }^{40} \mathrm{~K}$ aktivite konsantrasyonları $\mathrm{Bq} / \mathrm{kg}$ birimlerinde ölçülmüştür. Sağlık risklerini belirlemek için çeşitli çalışmalar yapılmıştır. Ayrıca bu çalışmalarda, UNSCEAR raporlarından elde edilen $(\mathrm{nGy} / \mathrm{h}) /(\mathrm{Bq} / \mathrm{kg})$ birimlerine göre dönüşüm faktörleri ve doğal radyonüklid konsantrasyonları kullanılarak malzemenin neden olduğu çeşitli tehlike indeksleri ve doz oranları değerleri hesaplanmıştır [5-18]. 
Bu çalışmada, Türkiye'nin Marmara Bölgesi'nden toplanan 7 adet mermer örneğinin doğal radyoaktivite seviyelerini belirlemek için ${ }^{226} \mathrm{Ra},{ }^{232} \mathrm{Th}$ ve ${ }^{40} \mathrm{~K}$ konsantrasyonları gama spektrometre sistemi ile ölçülmüştür. Bu çalışmanın diğer bir amacı, ölçülen aktivite konsantrasyonlarından yararlanarak soğrulan gama doz hızını (D), Yıllık Etkin Doz Eşdeğeri (YEDE), radyum eşdeğer aktivitesini (Raeq), iç tehlike endeksi (Hi) ve dış tehlike endeksi (Hd) parametreleri hesaplanarak radyolojik tehlikelerin araştırılmasıdır. Ayrıca, doğal olarak maruz kalınan radyasyonun sebep olduğu yaşam boyu kanser riski tespit edilmesi de amaçlanmıştır. Bu çalışmada üretilen veriler, bu alan için doğal radyoaktivite düzey veri tabanına katkıda bulunabilir.

\section{Deneysel çalışmalar}

Bu çalışmada, Türkiye Marmara Bölgesi'nde bulunan Balıkesir ilinden Marmara Adası, Marmara Gri, ve Yakamoz Kahve olmak üzere 3 adet, Bursa ilinden Crema Bello ve Rosalya Light olmak üzere 2 adet ve Bilecik ilinden Bilecik Rosalya ve Crema Nuova olmak üzere 2 adet, toplamda 7 mermer örneği toplanmıştır.

Toplanan örnekler ölçüm öncesinde bir dizi hazırlık aşamasından geçirilmiştir. Öncelikle örnekler ögütülerek toz haline getirilmiştir. Toz haline getirilen örnekler neminden arındırılmak amacıyla 24 saat boyunca $100{ }^{\circ} \mathrm{C}$ ' de kurutulmuştur. Kurutulmuş numuneler, herhangi bir gaz kaçağını engellemek için kapakları sıkıca kapatılmış kaplar içerisine doldurulmuştur.

${ }^{238}$ U'un bozunum zinciri içerisinde bulunan uzun yarı ömürlü ${ }^{226} \mathrm{Ra}$ ' nın yarı ömrü kendisinden daha kısa olan ${ }^{222} \mathrm{Rn}$ ile seküler bir denge içerisinde olması gerekmektedir. $\mathrm{Bu}$ nedenle hazırlanmış olan numunelerin ölçümlerine başlamadan önce seküler dengesinin sağlanabilmesi için 30 gün süreyle bekletilmiştir.

Mermer numunelerindeki ${ }^{226} \mathrm{Ra},{ }^{232} \mathrm{Th}$ ve ${ }^{40} \mathrm{~K}$ radyoaktivite konsantrasyonları Süleyman Demirel Üniversitesi Fen-Edebiyat Fakültesi Fizik bölümü Gama Spektroskopi laboratuarında bulunan Çok-Kanallı Analizöre (MCA) bağlı 3"x3" NaI (Tl) detektöründen oluşan bir gama spektrometresi kullanılarak ölçülmüştür $[19,20]$. Ölçümler sonucunda elde edilen spektrumlar MAESTRO-32 yazılımı kullanılarak analiz ediliştir. Ölçüm sisteminin şematik görünümü Şekil 1'de gösterilmiştir.

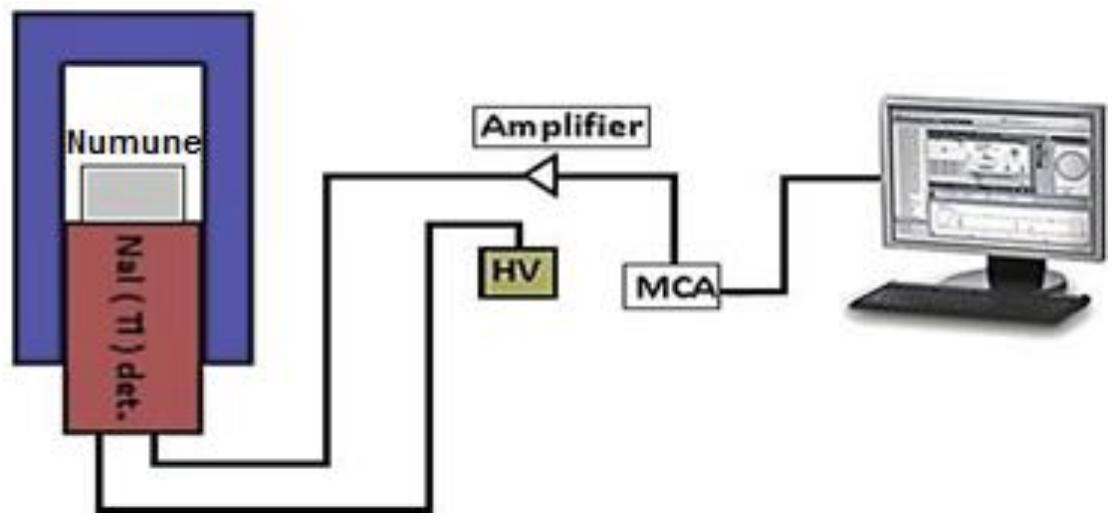

Şekil 1. Gama Spektrometre sistemi ve elektronik birimlerin şematik görünümü. 
Dedektörün algılama verimliliği kalibrasyonu için 6 farklı gama enerjisi kullanılarak yapılmıştır [21]. Elde edilen algılama verimliliği değerleri Şekil 2' de gösterilmiştir.

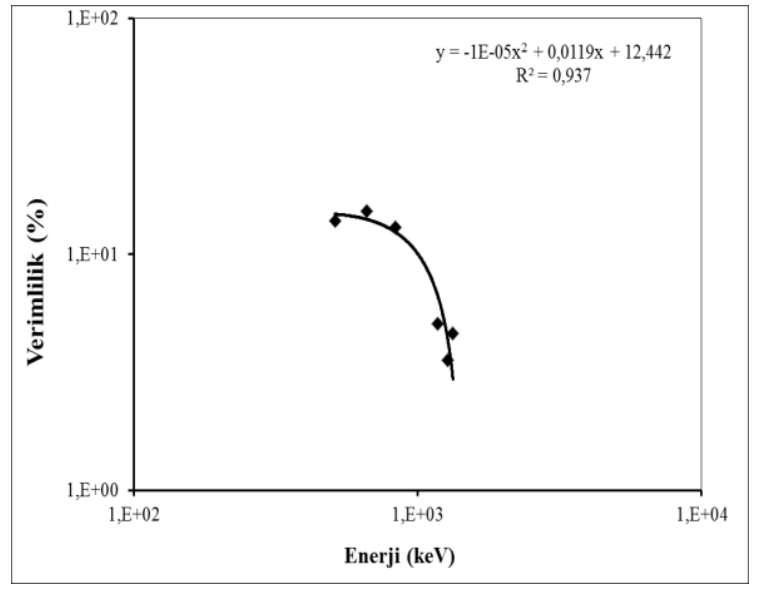

Şekil 2. Gama 1şını enerjilerinin bir fonksiyonu olarak algılama verimliliği.

Ölçümden önce sistemin enerji kalibrasyonunu ve algılama verimliliği kalibrasyonunu gerçekleştirmek gerekir. Enerji kalibrasyonu için $662 \mathrm{keV}$ enerjili $\gamma$-1şını yayınlayan ${ }^{137} \mathrm{Cs}$ ve 1173 ve $1332 \mathrm{keV}$ enerjili $\gamma$-1şını yayınlayan ${ }^{60} \mathrm{Co}$ radyoaktif kaynakları kullanılmıştır. Söz konusu kaynaklardan elde edilen gama 1şını spektrumu Şekil 3' de gösterilmektedir.

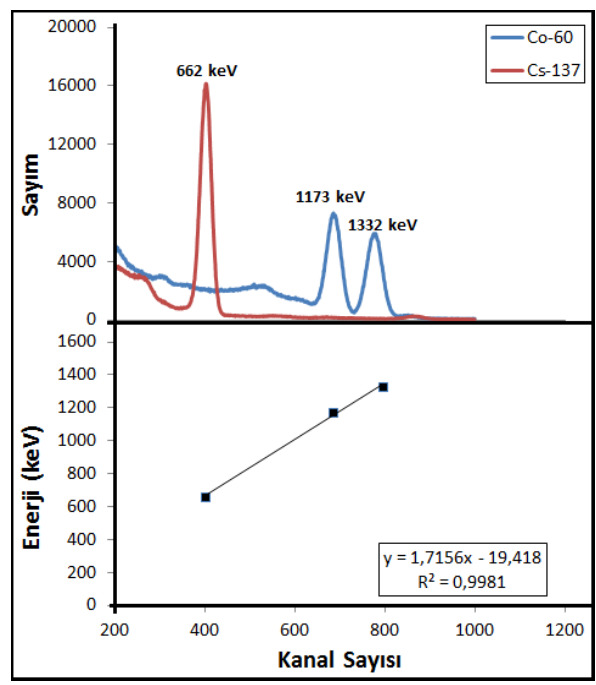

Şekil 3. ${ }^{137} \mathrm{Cs}$ ve ${ }^{60} \mathrm{Co}$ kaynaklarından elde edilen $\gamma$-1şını enerji spektrumu (üst) ve enerjiye (keV) karşılık gelen uygun kanal.

Mermer numunelerinin doğal radyonüklidlerin $\left({ }^{226} \mathrm{Ra},{ }^{232} \mathrm{Th}\right.$ ve $\left.{ }^{40} \mathrm{~K}\right)$ aktivite konsantrasyonlarını belirlemek için aşağıdaki bağıntı kullanılmıştır [15].

$C(B q / k g)=\frac{N_{S}-N_{B}}{E_{\gamma} \cdot P_{\gamma} \cdot t \cdot M_{S}}$ 
Burada, NS, numune için ölçülen net pik sayımlarını, NB, background için ölçülen net pik sayımlarını, E $\gamma$ spesifik $\gamma$-1şının detektör verimini, P $\gamma \gamma$-1şının emisyon olasılığını, $t$ sayım zamanını ve MS numunenin kütlesini $(\mathrm{kg})$ göstermektedir.

\section{Sonuçlar ve tartışma}

\subsection{Aktivite konsantrasyonu sonuçlart}

Türkiye'nin Marmara Bölgesi'ndeki bazı mermerlerde ${ }^{226} \mathrm{Ra},{ }^{232} \mathrm{Th}$ ve ${ }^{40} \mathrm{~K}$ aktivite konsantrasyonları ölçülmüştür. Ölçüm sonucunda elde edilen aktivite konsantrasyon sonuçlar1 ${ }^{226} \mathrm{Ra}$ için $25.342-48.835 \mathrm{~Bq} / \mathrm{kg},{ }^{232} \mathrm{Th}$ için $18.774-49.506 \mathrm{~Bq} / \mathrm{kg}$ ve ${ }^{40} \mathrm{~K}$ için $128.094-419.611 \mathrm{~Bq} / \mathrm{kg}$ arasında değişmektedir. Elde edilen aktivite konsantrasyonu sonuçları Şekil 4' de gösterilmiştir.

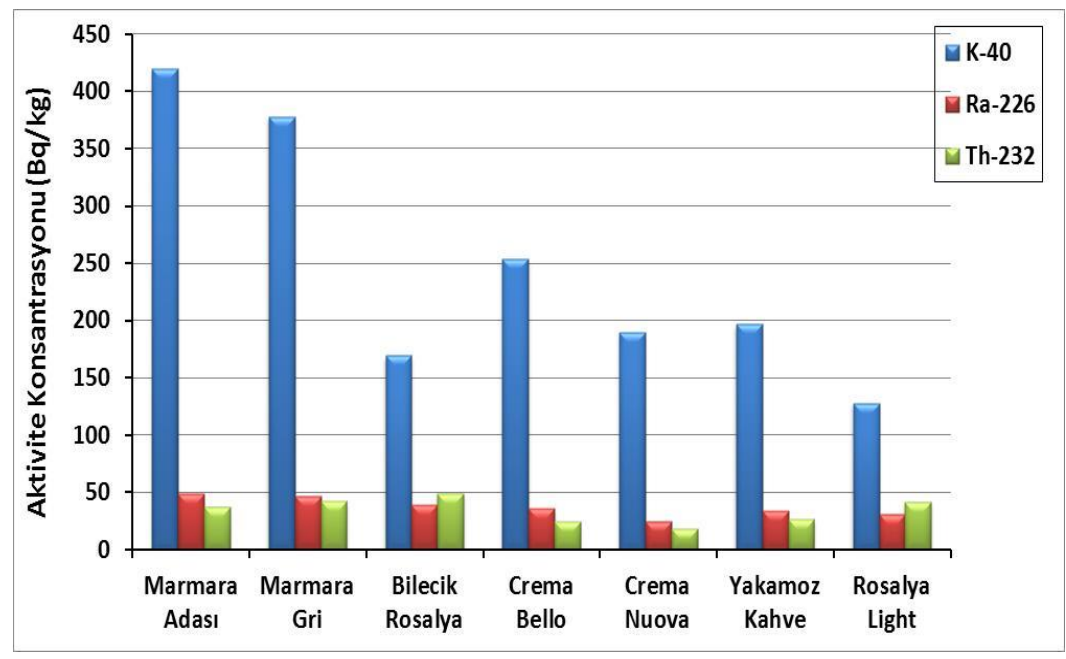

Şekil 4. Mermer numunelerinin aktivite konsantrasyonları.

Tablo 1'de gösterildiği gibi ölçülen ${ }^{226} \mathrm{Ra},{ }^{232} \mathrm{Th}$ ve ${ }^{40} \mathrm{~K}$ aktiviteleri, dünyanın farklı bölgelerindeki aktivite konsantrasyonları ve UNSCEAR 2000 raporlarında belirtilen dünya ortalaması ile karşılaştırılmıştır.

Tablo 1. Farklı bölgelerdeki aktiviteleri karşılaştırılması.

\begin{tabular}{lllll}
\hline Bölge & \multicolumn{3}{c}{ Aktivite Konsantrasyonları (Bq/kg) } & Referans \\
\cline { 2 - 4 } & ${ }^{\mathbf{2 2 6} \mathbf{R a}}$ & ${ }^{\mathbf{2 3 2} \mathbf{T h}}$ & ${ }^{\mathbf{4 0}} \mathbf{K}$ & \\
\hline Pakistan & 33 & 32 & 57 & {$[22]$} \\
Hindistan & 25,88 & 42,82 & 560,6 & {$[23]$} \\
Suudi Arabistan & 28,82 & 34,83 & 665,08 & {$[24]$} \\
Türkiye & 15,85 & 33,8 & 359 & {$[25]$} \\
Italya & 397 & 211 & 1265 & {$[26]$} \\
Misır & 137 & 82 & 1082 & {$[27]$} \\
Polonya & $31-122$ & $25-62$ & $320-1200$ & {$[28]$} \\
\hline
\end{tabular}


GÜNO $\breve{G L U K}$.

Tablo 1. (Devam1).

\begin{tabular}{lllll}
\hline Ürdün & 41,52 & 58,42 & 897 & {$[29]$} \\
Filistin & 71 & 82 & 780 & {$[30]$} \\
Iran & 77,4 & 44,5 & 1017,2 & {$[31]$} \\
Bu çalışma & $\mathbf{2 5 . 3 4 2}-\mathbf{4 8 . 8 3 5}$ & $\mathbf{1 8 . 7 7 4}-\mathbf{4 9 . 5 0 6}$ & $\mathbf{1 2 8 . 0 9 4}-\mathbf{4 1 9 . 6 1 1}$ & \\
Dünya Ortalaması & 50 & 50 & 500 & {$[3]$} \\
\hline
\end{tabular}

\subsection{Soğurulan gama doz hızı (D)}

Doğal olarak oluşan radyonüklitlerden $\left({ }^{226} \mathrm{Ra},{ }^{232} \mathrm{Th}\right.$ ve $\left.{ }^{40} \mathrm{~K}\right)$ gelen karasal $\gamma$-1şınları nedeniyle, zemin yüzeyinden $1 \mathrm{~m}$ yüksekliğinde havada soğrulan gama doz hızı, UNSCEAR, 2000 raporlarında verilen kurallara göre hesaplanmaktadır [3]. Bu kurallara göre, ${ }^{226} \mathrm{Ra}$ için $0.462 \mathrm{nGy} / \mathrm{saat},{ }^{232} \mathrm{Th}$ için $0.604 \mathrm{nGy} /$ saat ve ${ }^{40} \mathrm{~K}$ için 0.042 $\mathrm{nGy} /$ saat dönüşüm faktörleri birim aktivite konsantrasyonu başına $\mathrm{Bq} / \mathrm{kg}$ cinsinden havadaki soğrulan gama hızını hesaplamak için kullanılır. Buna göre, soğrulan gama doz hızı aşağıdaki bağıntı kullanılarak hesaplanabilir [3].

$D(n G y / h)=(0.462) C_{R a}+(0.604) C_{T h}+(0.0417) C_{K}$

Burada, $\mathrm{C}_{\mathrm{Ra}}, \mathrm{C}_{\mathrm{Th}}$ ve $\mathrm{C}_{\mathrm{K}}$ sirasiyla $226 \mathrm{Ra}, 232 \mathrm{Th}$ ve $40 \mathrm{~K} \mathrm{~Bq} / \mathrm{kg}$ cinsinden spesifik aktivitelerdir. Soğrulan gama doz hızı sonuçları, Şekil 5 'te gösterilmiştir.

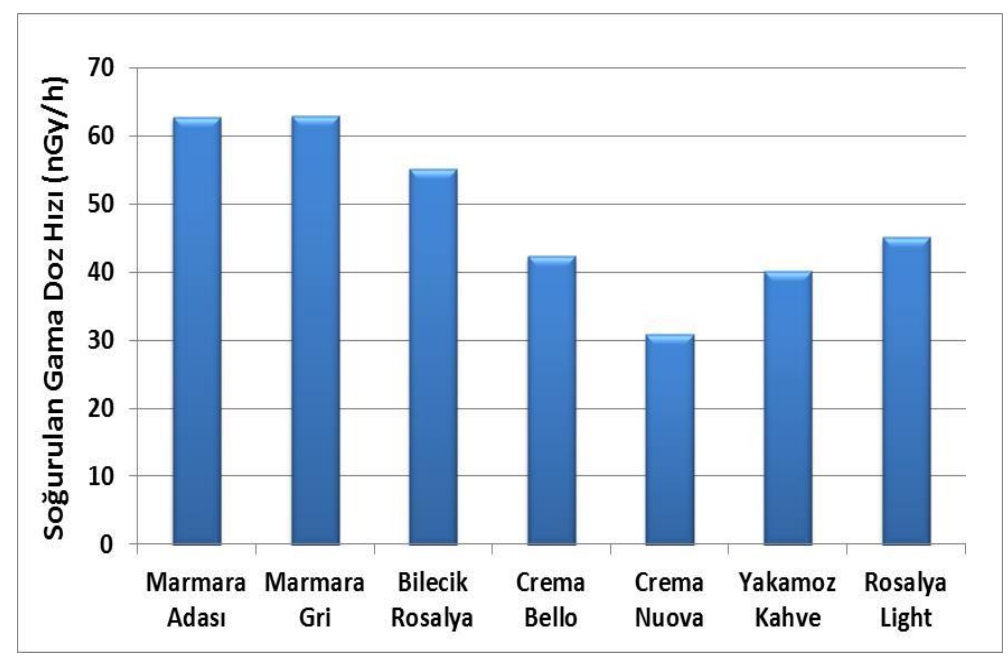

Şekil 5. Soğurulan gama doz hızı (D).

Şekil 5' den görüldüğü gibi soğrulan gama doz hızı için hesaplanan en düşük değer Bilecik' ten çıkarılan numunesinde, en yüksek değer Balıkesir' den çıkarılan Marmara Gri numunesinde bulunmuştur. Soğrulan gama doz hızı sonuçlarına bakıldığında Marmara Adası, Marmara Gri ve Bilecik Rosalya numunelerinden elde edilen sonuçlar UNSCEAR, 2000 raporlarında verilen ortalama değer olan $55 \mathrm{nGy} /$ saat ten yüksek çıktığ 1 ve diğer numunelerin bu ortalama değerden düşük olduğu görülmüşsür.

\subsection{Yıllık etkin doz eșdeğeri (YEDE)}

İnsanların yaşam alanları ve bu alanların çevresindeki sağlık etkilerinin belirlenmesi için yıllık etkin doz eşdeğeri (YEDE), soğrulan gama doz hızı kullanılarak hesaplanmaktadır. Yıllık etkin dozları hesaplamak için, soğrulan gama doz hızı, 
yetişkinler tarafından alınan etkin doz oranı ve zaman geçirme faktörü kullanılır. UNSCEAR2000 raporlarına göre, etkin doz dönüşüm katsayısı (DDF) 0.7 Sv / Gy ve zaman geçirme faktörü (ZGF) $0.2^{\prime}$ dir. Yıllık etkin doz eşdeğeri aşağıdaki denklemden hesaplanır [3]:

$$
Y E D E(m S v / y)=D(n G y / h) x D D F x Z G F x T
$$

Hesaplanan yıllık etkin doz eşdeğeri Şekil 6' da gösterilmiştir. Y1llık etkin doz eşdeğeri için elde edilen sonuçlar 0.038 ila $0.077 \mathrm{mSv} / \mathrm{y} 1 \mathrm{l}$ arasında değiştiği görülmüştür. Ayrıca, YEDE için bulunan sonuçların hiçbirinin belirtilen $0,460 \mathrm{mSv} / \mathrm{y}$ sınır değerini aşmadığı açıktır.

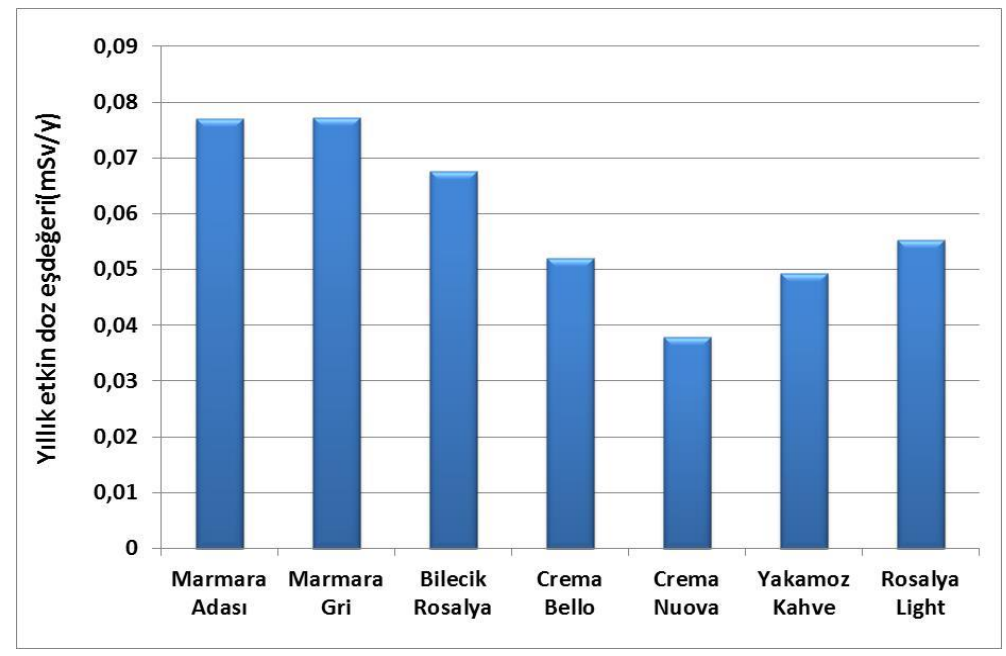

Şekil 6. Y1llık etkin doz eşdeğeri.

\subsection{Radyum eşdeğer aktivitesi $\left(R a_{e q}\right)$}

${ }^{226} \mathrm{Ra}$ ve bozunma ürünleri arasındaki radyoaktivite dengesizliğinden dolayı, materyallerdeki ${ }^{40} \mathrm{~K},{ }^{232} \mathrm{Th}$ ve ${ }^{226} \mathrm{Ra}$ gibi doğal radyoaktif nüklitler homojen olmadı̆̆ için materyallerde farklı miktarlarda bulunur. Radyum eşdeğer aktivitesi, doğal radyoaktif nüklitlerle ilgili radyasyon tehlikelerini dikkate alan ve materyaldeki aktivite konsantrasyonunu kontrol etmek için kullanılan bir hesaplama yöntemidir. Raeq için radyolojik sağlık güvenliği değerlendirmesi açısından izin verilen en yüksek değer 370 $\mathrm{Bq} / \mathrm{kg}$ 'dır [14]. Hesaplamada kullanılan denklem aşağıdaki gibi verilir [15]:

$$
\mathrm{Ra}_{\mathrm{eq}}=C_{R a}+(1,43) \cdot C_{T h}+(0,077) \cdot C_{K}
$$

Burada, $\mathrm{C}_{\mathrm{Ra}}, \mathrm{C}_{\mathrm{Th}}$ ve $\mathrm{C}_{\mathrm{K}}$ sirasiyla ${ }^{226} \mathrm{Ra},{ }^{232} \mathrm{Th}$ ve ${ }^{40} \mathrm{~K} \mathrm{~Bq} / \mathrm{kg}$ cinsinden spesifik aktivitelerdir. Radyum eşdeğer aktivitesi sonuçları, Şekil 7'de gösterilmiştir. 


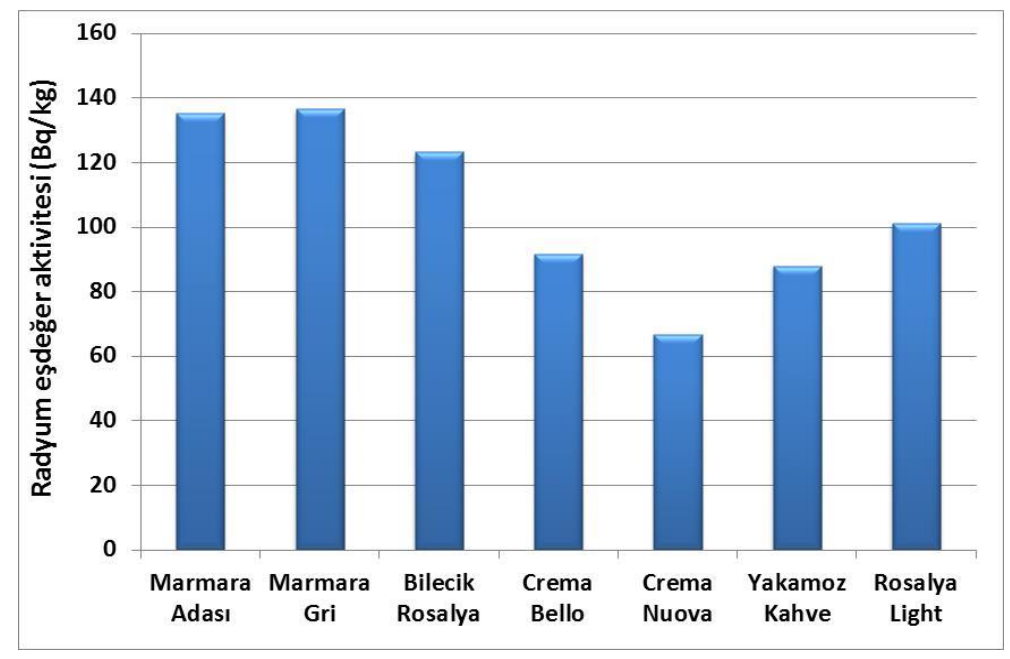

Şekil 7. Radyum eşdeğer aktivitesi.

Şekil 7'den görüldüğü gibi hesaplanan en yüksek radyum eşdeğer aktivitesi 136.575 $\mathrm{Bq} / \mathrm{kg}$ ile Marmara Gri numunesinde, en düşük değer ise Crema Nuova numunesinde bulunmuştur. $\mathrm{Ra}_{\mathrm{eq}}$ için radyolojik sağlık güvenliği değerlendirmesi açısından izin verilen en yüksek değer $370 \mathrm{~Bq} / \mathrm{kgg}^{\prime}$ dır [14]. Şekilden de görüldügüü gibi tüm numuneler çin elde edilen sonuçlar belirlenen limit değeri aşmamıştır.

\section{5. İç ve dış tehlike indeksi $(\mathrm{Hi}, \mathrm{Hd})$}

Primordial radyonüklid içeren materyallerin sağlık etkilerini değerlendirmek için kullanılan diğer bir parametre de İç (Hi) ve Diş (Hd) Tehlike İndeksi'dir. Hi ve Hd, radyasyon tehlikesinin göz ardı edilebilmesi için 1 sınırını aşmamalıdır. Hi ve Hd aşağıdaki denklemler kullanılarak hesaplanmıştır [18].

$$
\begin{aligned}
& H d=\left(\frac{1}{370}\right) \cdot C_{R a}+\left(\frac{1}{259}\right) \cdot C_{T h}+\left(\frac{1}{4810}\right) \cdot C_{K} \leq 1 \\
& H i=\left(\frac{1}{185}\right) \cdot C_{R a}+\left(\frac{1}{259}\right) \cdot C_{T h}+\left(\frac{1}{4810}\right) \cdot C_{K} \leq 1
\end{aligned}
$$

Burada, $\mathrm{C}_{\mathrm{Ra}}, \mathrm{C}_{\mathrm{Th}}$ ve $\mathrm{C}_{\mathrm{K}}$ sirasiyla ${ }^{226} \mathrm{Ra},{ }^{232} \mathrm{Th}$ ve ${ }^{40} \mathrm{~K} \mathrm{~Bq} / \mathrm{kg}$ cinsinden spesifik aktivitelerdir. İç ve Dış Tehlike İndeksi sonuçları, Şekil 8'de gösterilmiştir. 


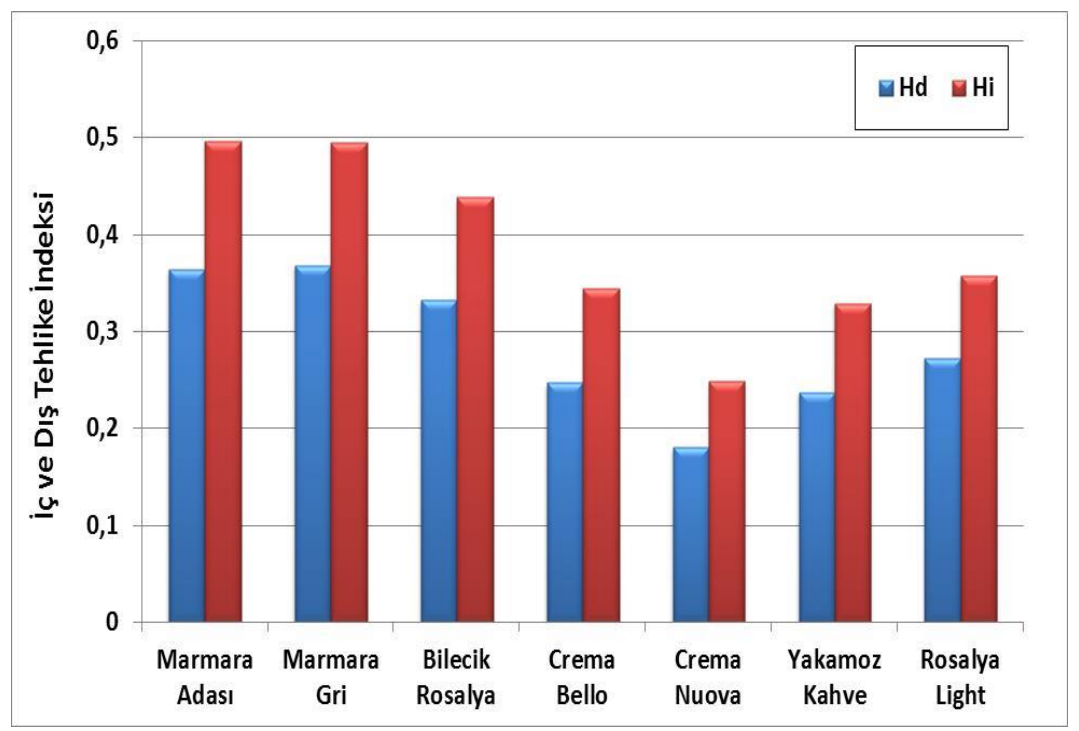

Şekil 8. İç ve diş tehlike indeksi.

Şekil 8' den görüldüğü gibi mermer numuneleri için hesaplanan iç ve diş tehlike indeksi değerlerinden tümü belirlenen 1 değerinden daha düşüktür.

\subsection{Yaşam boyu kanser riski (LCR)}

Yaşam boyu kanser riski (LCR), bir kişinin yaşamı boyunca belirli bir kanserojen veya radyasyon dozuna maruz kalması durumunda kanser gelişmesi olasılığı olarak tanımlanır.

Yaşam boyu kanser riski hesaplanırken insanın ortalama yaşam süresi 70 yıl olarak kabul edilmektedir. Buna göre, Yaşam boyu kanser riski (LCR) aşağıdaki denklem kullanılarak hesaplanmaktadır [32].

$L C R=Y E D E \times Y S \times R F$

Burada, YEDE denklem (3) ile hesaplanan yıllık etkin dozdur, YS bir insan için ortalama yaşam süresi (yaklaşık 70 yıl olduğu varsayılmaktadır) ve RF insanlar için ölümcül bir kanser risk faktörüdür ve ICRP60 raporlarına göre bu değer $0.05 \mathrm{~Sv}^{-1}$ olarak verilmiştir [31]. Yaşam boyu kanser riski (LCR) için elde edilen sonuçlar Şekil 9' da gösterilmiştir. 


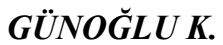

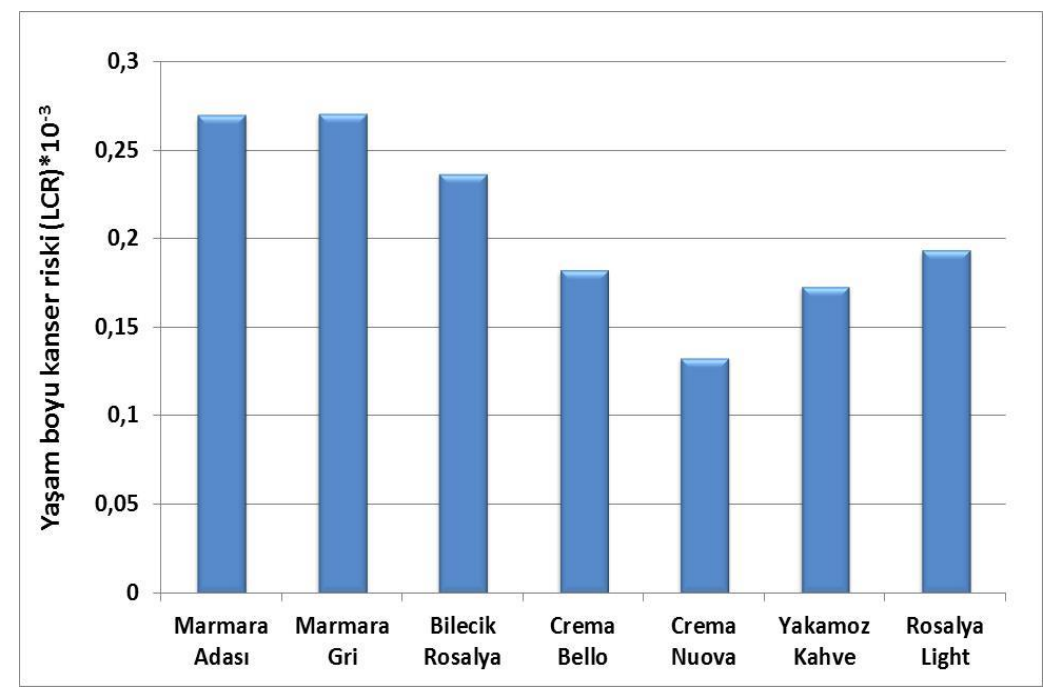

Şekil 9. Yaşam boyu kanser riski (LCR).

Yaşam boyu kanser riski (LCR) için limit değer $0.290 \times 10^{-3}$ olarak verilmiştir. Şekil 9' da verilen sonuçlar verilen bu limit değerden düşük olduğunu göstermektedir.

Aktivite konsantrasyonları kullanılarak elde doz oranları ve radyolojik indeks sayısal verileri Tablo 2' de gösterilmiştir.

Tablo 2. Doz oranları ve radyolojik indeks sayısal verileri.

\begin{tabular}{ccccccc}
\hline \multirow{2}{*}{ Numune adı } & \multicolumn{6}{c}{ Doz Oranları ve Radyolojik Tehlike İndeksleri } \\
\cline { 2 - 7 } & $\mathbf{D}(\mathbf{n G y} / \mathbf{h})$ & YEDE $(\mathbf{m S v} / \mathbf{y})$ & $\mathbf{R a e q}(\mathbf{B q} / \mathbf{k g})$ & $\mathbf{H}_{\mathbf{d}}$ & $\mathbf{H}_{\mathbf{i}}$ & ELCR*10-3$^{-3}$ \\
\hline Marmara Adası & 62,83 & 0,077 & 135,060 & 0,365 & 0,497 & 0,270 \\
Marmara Gri & 63,02 & 0,077 & 136,575 & 0,369 & 0,496 & 0,271 \\
Bilecik Rosalya & 55,12 & 0,068 & 123,140 & 0,333 & 0,439 & 0,237 \\
Crema Bello & 42,45 & 0,052 & 91,599 & 0,247 & 0,345 & 0,182 \\
Crema Nuova & 30,95 & 0,038 & 66,782 & 0,180 & 0,249 & 0,133 \\
Yakamoz Kahve & 40,27 & 0,049 & 87,862 & 0,237 & 0,329 & 0,173 \\
Rosalya Light & 45,11 & 0,055 & 101,076 & 0,273 & 0,358 & 0,194 \\
\hline Ortalama \pm S.H & $45,34 \pm 6,87$ & $0,056 \pm 0,01$ & $96,756 \pm 15,07$ & $0,261 \pm 0,04$ & $0,347 \pm 0,05$ & $0,195 \pm 0,029$ \\
\hline
\end{tabular}

\section{Tartışma}

Marmara Bölgesi'nden çıkarılan bazı mermer örneklerindeki ${ }^{226} \mathrm{Ra},{ }^{232} \mathrm{Th}$ ve ${ }^{40} \mathrm{~K}$ doğal radyonüklitlerin aktivite konsantrasyonları $\mathrm{NaI}(\mathrm{Tl})$ detektörü ile gama 1 şını spektroskopisi tekniği kullanılarak ölçülmüştür. Mermer numunelerindeki ${ }^{226} \mathrm{Ra},{ }^{232} \mathrm{Th}$ ve ${ }^{40} \mathrm{~K}$ ortalama aktivite konsantrasyonları, sirasiyla $37.446,34.593$ ve $248.041 \mathrm{~Bq} / \mathrm{kg}$ olarak ölçülmüştür. Sonuçlar, mermer numunelerinde doğal olarak radyoaktif element içeriğine bağlı radyasyon dozlarının değerlendirilmesi için yararlıdır. Mermer örneklerinde deneysel olarak elde edilen radyonüklidlerin konsantrasyonları kullanılarak absorbe edilen gama doz oranı, yıllık etkili doz eşdeğeri, aşırı yaşam boyu kanser riski, radyum eşdeğeri aktiviteleri, harici tehlike indeksleri, içsel tehlike indeksleri ve gama indeks değerleri hesaplanmıştır. Hesaplanan sonuçlar belirlenmiş 
olan sınır değerlerin altında olduğu görülmüştür. Elde edilen sonuçlar 1şığında, incelenen mermer örneklerinin radyolojik açıdan bir zarar teşkil etmediği söylenebilir.

\section{Kaynaklar}

[1] Malain, D., Regan, P.H., Bradley, D.A., Matthews, M., Al-Sulaiti, H.A., Santawamaitre, T. An evaluation of the natural radioactivity in Andaman beach sand samples of Thailand after the 2004 tsunami, Appl. Radiat. Isot., 70(8), 1467-1474, (2012).

[2] Veiga, R., Sanches, N., Anjos, R.M., Macario, K., Bastos, J., Iguatemy, M., Aguiar, J.G., Santos, A.M.A., Mosquera, B., Carvalho, C., Filho, M.B., Umisedo, N.K., Measurement of natural radioactivity in Brazilian beach sands. Radiat. Meas., 41(2), 189-196, (2006).

[3] UNSCEAR. Sources and Effects of Ionizing radiation, UnitedNations Scientific Committee on the Effects of Atomic radiation. Exposures from Natural Radiation Sources, Annex B, UnitedNations, New York (2000).

[4] Stoulos, S., Manolopoulou, M., Papastefanou, C., Assessment of natural radiation exposure and radon exhalation from building materials in Greece, $\mathbf{J}$. Environ. Radioact., 69, 225-240, (2003).

[5] Abbady, A.G.E., Uosif, M.A.M., El-Taher, A., Natural radioactivityand dose assessment for phosphate rocks from Wadi El-Mashashand El-Mahamid Mines, Egypt, J. Environ. Radioact., 84,65 (2005).

[6] El Afifi, E.M., Hilal, M.A., Khalifa, S.M., Aly, H.F., Evaluation of U, Th, K and Emanated radonin some NORM and TENORM, Radiat. Meas., 41, 627 (2006).

[7] Al-Jarallah, M.I., Fazal-ur-Rehman, Musazay, M.S., Aksoy, A., Correlation between radon exhalation and radium content in granite samples used as construction material in Saudi Arabia, Radiat.Meas., 40, 625 (2005).

[8] Uyanik, N.A., Öncü, Z., Uyanik, O., Bozcu, M., Akkurt, İ., Günoğlu, K., Yağmurlu, F., Distribution of natural radioactivity from $40 \mathrm{~K}$ radioelement in volcanics of Sandiklı-Şuhut (Afyon) area, Acta Physica Polonica A, 128(2-B), 438-440, (2012).

[9] Canbaz, B., Füsun Çam, N., Yaprak, G., Candan, O., Natural radioactivity (226Ra,232Th and40K) and assessment of radiologicalhazards in the Kestanbolgranitoid, Turkey, Radiat. Prot. Dosim.,141, 192 (2010).

[10] Fazal-ur-Rehman, M.I. Al-Jarallah, M.S. Musazay, F. Abu-Jarad,. Application of the can technique and radon gas analyzer for radonexhalation measurements, Appl. Radiat. Isot. 59, 353 (2003).

[11] Myatt, T.A., Allen, J.G., Minegishi, T., McCarthy, W.B., Mac-Intosh, D.L., McCarthy, J.F., Assessing exposure to granite countertopsPart 1: radiation, J. Expo. Sci. Environ. Epidemiol, 20, 280 (2010).

[12] Al-Selah, F.S., Al-Berzan, B., Measurements of natural radioac-tivity in some kinds of marble and granite used in Riyadh region, J. Nucl. Radiat. Phys., 2, 25-36, (2007).

[13] El-Dine, N.W., El-Shershaby, A., Ahmed, F., Abdel-Haleem, A.S., Measurement of radioactivity and radon exhalation ratein different kinds of marbles and granites, Appl. Radiat. Isot., 55, 853, (2001).

[14] Beretka, J., Mathew, P.J., Natural radioactivity of Australian building materials, industrial wastes and by-products, Health Phys., 48, 87-95 (1985). 
[15] Akkurt I. ve Gunoglu, K., Natural radioactivity measurements and radiation dose estimation in some sedimentary rock samples in Turkey, Science and Technology of Nuclear Installations, Volume 2014 (2014).

[16] Kohshi, C., Takao, I., Hideo, S., Terrestrial gamma radiation in Koshi prefecture, Japan, J. Health Sci., 47, 362 (2001).

[17] Akkurt, I., Uyanik, N.A., Gunoglu, K., Radiation dose estimation: an in vitro measurement for Isparta-Turkey IJCESEN, 1(1),1-4, (2015).

[18] Günay, O., Saç, M.M., Içhedef, M. Taşköprü, C. Soil gas radon concentrations along the Ganos Fault (GF), Arabian Journal of Geoscience, 11:213. Print ISSN: 1866-7538, (2018)

[19] Akkurt I. Effective atomic and electron numbers of some steels at different energies. Annals of Nuclear Energy, 36, 1702-1705, (2009).

[20] Akkurt I., Basyigit, C., Kilincarslan, S., Mavi, B., Akkurt, A. Radiation shielding of concretes containing different aggregates, Cement \& Concrete Composites, 28, 153-157, (2006).

[21] Akkurt, I., Gunoglu, K. and Arda, S.S., Detection efficiency of NaI(Tl) detector in 511-1332 keV energy range, Science and Technology of Nuclear Installations, 1-5, (2014).

[22] Iqbal, M., Tufail, M., ve Mirza, S.M., Measurement of natural radioactivity in marble found in Pakistan using a $\mathrm{NaI}(\mathrm{Tl})$ gamma-ray spectrometer. Journal of Environmental Radioactivity, 51, 255-265, (2000).

[23] Senthilkumar, G., Raghu, Y., Sivakumar, S., Chandrasekaran, A., Anand, D. P., \& Ravisankar, R., Natural radioactivity measurement and evaluation of radiological hazards in some commercial flooring materials used in Thiruvannamalai, Tamilnadu, India, Journal of Radiation Research and Applied Sciences, 7, 1, 116-122, (2014).

[24] Al-Zahrani, J.H. Estimation of natural radioactivity in local and imported polished granite used as building materials in Saudi Arabia, Journal of Radiation Research and Applied Sciences, 10, 241-245, (2017)

[25] Osmanlioglu, A.E., Natural radioactivity and evaluation of effective dose equivalent of granites in Turkey, Radiation Protection Dosimetry, 121, 325329, (2006).

[26] Sesana, L., Fumagalli, M., Carnevale, M., Polla, G., Facchini, U., Colombo, A., Tunesi, A., De Capitani, L., Rusconi, R., Natural radionuclides in the rocks of the Valle del Carvo Pluto in Piedmon, Radiation Protection Dosimetry, 118, 3, 337-344 (2006).

[27] Amin, R.M., Gamma radiation measurements of naturally occurring radioactive samples from Egyptian commercial granites, Environmental Earth Sciences, 67(3), 771-775, (2012).

[28] Malczewski, M., Teper, L., \& Dorda, J., Assessment of natural andanthropogenic radioactivity levels in rocks and soils in the environs of Swieradow Zdrojin Sudetes, Poland, by in situ gamma-ray spectrometry, Journal of Environmental Radioactivity, 73, 233-245, (2004).

[29] Sharaf, J.M., \& Hamideen, M.S., Measurement of natural radioactivity in Jordanian building materials and their contribution to the public indoor gamma dose rate, Applied Radiation and Isotopes, 80, 61-66, (2013).

[30] Thabayneh, K. M., Measurement of natural radioactivity and radon exhala- tion rate in granite samples used in Palestinian buildings, Arabian Journal for Science and Engineering, 1-7, (2013). 
[31] Günay O., Determination of natural radioactivity and radiological effects in some soil samples in Beykoz-Istanbul, European Journal of Science and Technology 12, 9-14, (2018).

[32] Abbasi, A., Calculation of gamma radiation dose rate and radon concentra- tion due to granites used as building materials in Iran, Radiation Protection Dosimetry, 155(3), 335-342, (2013). 